\title{
Nucleon mass radii and distribution: Holographic QCD, lattice QCD, and GlueX data
}

\author{
Kiminad A. Mamo* \\ Physics Division, Argonne National Laboratory, Argonne, Illinois 60439, USA \\ Ismail Zahed ${ }^{\dagger}$ \\ Center for Nuclear Theory, Department of Physics and Astronomy, Stony Brook University, \\ Stony Brook, New York 11794-3800, USA
}

(Received 12 March 2021; accepted 15 April 2021; published 13 May 2021)

\begin{abstract}
We briefly review and expand our recent analysis for all three invariant $A, B, D$ gravitational form factors of the nucleon in holographic QCD. They compare well to the gluonic gravitational form factors recently measured using lattice QCD simulations. The holographic $A$-term is fixed by the tensor $T=2^{++}$(graviton) Regge trajectory, and the $D$-term by the difference between the tensor $T=2^{++}$(graviton) and scalar $S=0^{++}$(dilaton) Regge trajectories. The $B$-term is null in the absence of a tensor coupling to a Dirac fermion in bulk. A first measurement of the tensor form factor $A$-term is already accessible using the current GlueX data, and therefore the tensor gluonic mass radius, pressure, and shear inside the proton, thanks to holography. The holographic $A$-term and $D$-term can be expressed exactly in terms of harmonic numbers. The tensor mass radius from the holographic threshold is found to be $\left\langle r_{G T}^{2}\right\rangle \approx(0.57-0.60 \mathrm{fm})^{2}$, in agreement with $\left\langle r_{G T}^{2}\right\rangle \approx(0.62 \mathrm{fm})^{2}$ as extracted from the overall numerical lattice data, and empirical GlueX data. The scalar mass radius is found to be slightly larger $\left\langle r_{G S}^{2}\right\rangle \approx(0.7 \mathrm{fm})^{2}$.
\end{abstract}

DOI: 10.1103/PhysRevD.103.094010

\section{INTRODUCTION}

A persistent and fundamental question in physics is about the origin of mass in the nucleon, and therefore in all visible hadronic mass in the Universe. Where does it come from, and how is it distributed inside the nucleon? Unlike the Higgs in electroweak theory which is at the origin of the leptonic and current quark masses, most of the hadronic mass of the visible Universe stems from QCD, a theory with almost no mass [1,2] (and references therein).

The dual quantum breaking of conformal symmetry and the spontaneous breaking of chiral symmetry in QCD are at the origin of this mass without mass. These two fundamental phenomena are tied by strong topological fluctuations in the QCD vacuum: eternal tunneling events between gauge vacua with different winding numbers, also known as instantons and anti-instantons [3,4] (and references therein).

While decisive understanding of these two phenomena has been achieved theoretically and numerically using

\footnotetext{
*kmamo@anl.gov

ismail.zahed@stonybrook.edu
}

Published by the American Physical Society under the terms of the Creative Commons Attribution 4.0 International license. Further distribution of this work must maintain attribution to the author(s) and the published article's title, journal citation, and DOI. Funded by SCOAP . lattice QCD simulations [5,6], empirical measurements to support this understanding is only now emerging at current and dedicated electron machines [7-10]. Recently, the GlueX Collaboration [8] at JLAB has reported threshold data for photoproduction of charmonium $J / \Psi$ that may start to lift the lid on some of these fundamental questions. Indeed, near threshold the elastic production of a heavy vector meson is likely to proceed mostly through gluons or more precisely tensor glueballs, as is the case way above threshold through the strong Pomeron exchange in the diffractive regime [11,12].

In a recent analysis of the GlueX data using a holographic construction, we have shown [13] that the threshold differential cross section is only sensitive to the tensor gravitational form factor, and suggested that this tensor form factor or $A$-term is extractable from the current data under a minimal but universal set of holographic assumptions. This allows for a first extraction of the tensor mass radius among other things. Remarkably, the holographic construction ties the $A$ - and $D$-gravitational form factors, thereby allowing for the extraction of the gluonic pressure and shear inside the proton. For completeness, we note the holographic discussion regarding the extraction of the gluon condensate in the proton using the GlueX data in [14].

In Sec. II we briefly review and expand our arguments for the holographic $A, B, D$ invariant gravitational form 
factors for the proton, extract the tensor mass radius, and compare the results to the most recent lattice data. In Sec. III we use the holographic relationship between the $A$ - and $D$-term to analyze the gluonic pressure and shear inside the proton. The pressure inside the proton results from a delicate balance between the repulsive tensor glueball contribution at short distances, and the attractive scalar glueball contribution at large distances. An estimate of the scalar mass radius is made. In Sec. IV we show how to use the current GlueX data to extract empirically the $A$-form factor. The result is in remarkable agreement with the holographic result and lattice data. Our conclusions are in Sec. V.

\section{GRAVITATIONAL FORM FACTORS}

The standard decomposition of the energy-momentum form factor in a nucleon state is [15-18]

$$
\begin{aligned}
\left\langle p_{2}\left|T^{\mu \nu}(0)\right| p_{1}\right\rangle= & \bar{u}\left(p_{2}\right)\left(A(k) \gamma^{(\mu} p^{\nu)}+B(k) \frac{i p^{(\mu} \sigma^{\nu) \alpha} k_{\alpha}}{2 m_{N}}\right. \\
& \left.+C(k) \frac{k^{\mu} k^{\nu}-\eta^{\mu \nu} k^{2}}{m_{N}}\right) u\left(p_{1}\right)
\end{aligned}
$$

with $\left.\quad a^{(\mu} b^{\nu}\right)=\frac{1}{2}\left(a^{\mu} b^{\nu}+a^{\nu} b^{\mu}\right), \quad k^{2}=\left(p_{2}-p_{1}\right)^{2}=t$, $p=\left(p_{1}+p_{2}\right) / 2$, and the normalization $\bar{u} u=2 m_{N}$. Equation (5) is conserved and traceful. Throughout, $D(k)=4 C(k)$ will be used interchangeably. In holography, Eq. (1) sources the metric fluctuations in bulk,

$$
g_{M N}(z) \rightarrow g_{M N}(z)+h_{M N}(x, z)
$$

with line element $d s^{2}=g_{M N}(z) d x^{M} d x^{N}$ in a fivedimensional anti-de Sitter space or $\mathrm{AdS}_{5}$, in the double limit of large $N_{c}$ and strong gauge coupling [19] (and references therein). The form factors in (1) follow from the coupling of the irreducible representations of the metric fluctuations $h_{\mu \nu}$, to a bulk Dirac fermion with chiral components $\psi_{L, R}$. The bulk metric fluctuations can be decomposed in terms of the $2 \oplus 1 \oplus 0$ invariant tensors [20]

$$
\begin{aligned}
h_{\mu \nu}(k, z)= & {\left[\epsilon_{\mu \nu}^{T T} h(k, z)+k_{\mu} k_{\nu} H(k, z)\right] } \\
& +\left[k_{\mu} A_{\nu}^{\perp}(k, z)+k_{\nu} A_{\mu}^{\perp}(k, z)\right] \\
& +\left[\frac{1}{3} \eta_{\mu \nu} f(k, z)\right],
\end{aligned}
$$

which is the spin-2 made of the transverse-traceless part $h$ plus the longitudinal-traceful part $H$, the spin- 1 made of the transverse vector $A_{\mu}^{\perp}$, and the spin-0 traceful part $f$.

\section{A. $A$-term}

To determine the $A$-term, we contract the energymomentum form factor (1) with a spin-2 transversetraceless polarization tensor $\epsilon_{\mu \nu}^{T T}$,

$$
\begin{aligned}
\left\langle p_{2}\left|\epsilon_{\mu \nu}^{T T} T^{\mu \nu}(0)\right| p_{1}\right\rangle & =\bar{u}\left(p_{2}\right)\left(A(k) \epsilon_{\mu \nu}^{T T} \gamma^{\mu} p^{\nu}\right) u\left(p_{1}\right) \\
& =\bar{u}\left(p_{2}\right) \frac{\delta}{\delta h_{0}}\left(\frac{\tilde{A}}{2} \int d z \sqrt{g} e^{-\phi(z)} z\left(\psi_{R}^{2}(z)+\psi_{L}^{2}(z)\right) h(k, z) \times \epsilon_{\mu \nu}^{T T} \gamma^{\mu} p^{\nu}\right) u\left(p_{1}\right) \\
& =\bar{u}\left(p_{2}\right)\left(\frac{\tilde{A}}{2} \int d z \sqrt{g} e^{-\phi(z)} z\left(\psi_{R}^{2}(z)+\psi_{L}^{2}(z)\right) \chi(k, z) \times \epsilon_{\mu \nu}^{T T} \gamma^{\mu} p^{\nu}\right) u\left(p_{1}\right) .
\end{aligned}
$$

The last two lines follow from a tree level Witten diagram and the holographic dictionary in the soft wall construction as detailed in $[13,19,21]$. They correspond to the coupling of the transverse-traceless part of the graviton $h(k, z)=h_{0} \chi(k, z)$ with $\chi(k, 0)=1$ (dual to $2^{++}$tensor glueballs) to a Dirac fermion in bulk. More specifically [13] ${ }^{1}$

$$
\begin{aligned}
A(k) & =\frac{\tilde{A}}{2} \int d z \sqrt{g} e^{-\phi(z)} z\left(\psi_{R}^{2}(z)+\psi_{L}^{2}(z)\right) \chi(k, z) \\
& =\tilde{A}\left(\left(1-2 a_{k}\right)\left(1+a_{k}^{2}\right)+a_{k}\left(1+a_{k}\right)\left(1+2 a_{k}^{2}\right)\left(H\left(\frac{1+a_{k}}{2}\right)-H\left(\frac{a_{k}}{2}\right)\right)\right)
\end{aligned}
$$

with $a_{k}=-k^{2} / 8 \kappa_{N}^{2}$. Here $H(x)=\psi(1+x)+\gamma$ is the harmonic number or digamma function plus Euler number. The scale $\kappa_{N}$ follows from the dilaton profile $\phi(z)=\kappa_{N}^{2} z^{2}$. It is dual to the string tension in QCD. $A(0)$ is not fixed in holography (1-point function). We have defined $\tilde{A}=A(0)$ to encode $\mathcal{O}\left(1 / N_{c}\right)$ corrections coming from 1-loop and higher Witten diagrams for the transverse-traceless tensor part of the energy momentum tensor. We have checked that (5) is in numerical agreement with a result in [21] modulo the overall normalization.

\footnotetext{
${ }^{1}$ In [13] there is a typo in the argument of the digamma function $\psi(x) \rightarrow \psi(1+x)$.
} 


\section{B. $D$-term}

To determine the $C$-term or $D$-term $(D=4 C)$, we contract the energy-momentum form factor (1) with $\frac{1}{3} \eta_{\mu \nu}$,

$$
\begin{aligned}
\frac{1}{3}\left\langle p_{2}\left|\eta_{\mu \nu} T^{\mu \nu}(0)\right| p_{1}\right\rangle & =\bar{u}\left(p_{2}\right)\left(A(k) \frac{m_{N}}{3}+\frac{k^{2}}{12 m_{N}} B(k)-\frac{k^{2}}{m_{N}} C(k)\right) u\left(p_{1}\right) \\
& =\bar{u}\left(p_{2}\right) \frac{\delta}{\delta f_{0}}\left(\frac{\tilde{C}}{2} \int d z \sqrt{g} e^{-\phi(z)} z\left(\psi_{R}^{2}(z)+\psi_{L}^{2}(z)\right) f(k, z) \times \frac{1}{3} \eta_{\mu \nu} \gamma^{\mu} p^{\nu}\right) u\left(p_{1}\right) \\
& =\bar{u}\left(p_{2}\right)\left(\frac{\tilde{C}}{2} \int d z \sqrt{g} e^{-\phi(z)} z\left(\psi_{R}^{2}(z)+\psi_{L}^{2}(z)\right) \chi(k, z) \times \frac{m_{N}}{3}\right) u\left(p_{1}\right) \\
& =\bar{u}\left(p_{2}\right)\left(A_{S}(k) \frac{m_{N}}{3}\right) u\left(p_{1}\right)
\end{aligned}
$$

where the constant $A_{S}(0)=\tilde{C}$ encodes the $\mathcal{O}\left(1 / N_{c}\right)$ corrections coming from 1-loop and higher Witten diagrams for the traceful part of the energy momentum tensor. In the line before last in (6) we used the coupling of the traceful part of the graviton or dilaton $f(k, z)=f_{0}-$ $4 \phi_{0}+4 \phi_{0} \chi(k, z)$ with $f_{0}=4 \phi_{0}$ [20] (dual to $S=0^{++}$ scalar glueballs) to a Dirac fermion in bulk, and again a tree level Witten diagram and the holographic dictionary as detailed in $[13,19,21]$. More specifically ${ }^{2}$

$$
\begin{aligned}
C(k) & =\frac{1}{3} \frac{m_{N}^{2}}{k^{2}}\left(A(k)-A_{S}(k)\right)+\frac{1}{12} B(k) \\
& \rightarrow \frac{1}{3} \frac{m_{N}^{2}}{k^{2}}\left(A(k)-A_{S}(k)\right)
\end{aligned}
$$

with $A_{S}(k) / A(k)=\tilde{C} / \tilde{A}$, as the $T=2^{++}$and $S=0^{++}$ glueballs are degenerate in the present soft wall construction at large $N_{c}$ (they have the same anomalous dimension $\left.\Delta_{T, S}=4\right)$. Since the Pauli-like form factor $B(k)=0$, as the coupling of the graviton to the bulk Dirac fermion through the spin-connection vanishes, the rightmost result follows.

The absence of massless modes in the scalar channel requires that $\tilde{C}=\tilde{A}$. This can also be checked by inserting (7) in (1) and tracing, so that $\left\langle p\left|T_{\mu}^{\mu}(0)\right| p\right\rangle=2 \tilde{C} m_{N}^{2}$ in any frame. Similarly, the 00-component of the energy momentum tensor gives $\left\langle p\left|T^{00}(0)\right| p\right\rangle=2 \tilde{A} p_{0}^{2} \rightarrow 2 \tilde{A} m_{N}^{2}$, with the rightmost result following in the rest frame only. Both results are expected from Poincaré symmetry $\left\langle p\left|T^{\mu \nu}(0)\right| p\right\rangle=$ $2 p^{\mu} p^{\nu}$. Therefore $\tilde{A}=\tilde{C}$ can be identified (they are 1 in full QCD, but less than 1 in the holographic limit of QCD which is gluonic in leading order in $1 / N_{c}$ ) and (7) vanishes.

At finite $N_{c}$, the anomalous dimensions are not equal with $\Delta_{T} \neq \Delta_{S} \neq 4$, e.g., [22]

\footnotetext{
${ }^{2}$ In [13] $C(k)$ was taken to be sourced by the scalar bulk field $f(k, z)$. Due to mixing of glueball fields in bulk this is an approximation. In fact, $f(k, z)$ sources exactly the scalar dilaton field $T_{\mu}^{\mu}$ as we now have shown.
}

$$
\Delta_{S} \rightarrow 4+\left(\beta^{\prime}\left(\alpha_{s}\right)-\frac{2}{\alpha_{s}} \beta\left(\alpha_{s}\right)\right)
$$

corrected by the beta function $\beta\left(\alpha_{s}\right)$, and $C(k)$ does not vanish in general. Only $A(0)=A_{S}(0)=1$ is required by the absence of a massless pole and Poincaré symmetry. Note that since $m_{S}<m_{T}$, the form factor $C(k)$ is in general negative. Also note that the $S=0^{++}$glueball is expected to mix strongly with the scalar sigma meson at large but finite $1 / N_{c}$.

\section{Gluonic gravitational radii}

The tensor gravitational radius following from (1) is $\left(k^{2}=t=-K^{2}\right)$

$$
\left\langle r_{G T}^{2}\right\rangle=-6\left(\frac{d \operatorname{Ln} A(K)}{d K^{2}}\right)_{0}=\left(2-H\left(\frac{1}{2}\right)\right) \frac{3}{4 \kappa_{N}^{2}}=\frac{1.04}{\kappa_{N}^{2}} .
$$

The $T=2^{++}$tensor and $0^{++}$scalar glueballs map to a graviton and dilaton dual to $T^{\mu \nu}$ and $T_{\mu}^{\mu}$, respectively, in bulk, with anomalous dimensions $\Delta_{T, S}=$ four- and fivedimensional squared masses $m_{5, T, S}^{2}=\Delta_{T, S}\left(\Delta_{T, S}-4\right)=0$, with Regge trajectories given by [23-25]

$$
m_{T, S}^{2}(n)=8 \kappa_{N}^{2}(n+2) .
$$

The slope is related to half the slope of the nucleon (twist $\tau_{N}=3$ ) and rho meson radial Regge trajectories

$m_{N}^{2}(n)=4 \kappa_{N}^{2}\left(n+\tau_{N}-1\right), \quad m_{\rho}^{2}(n)=4 \kappa_{N}^{2}(n+1)$.

Recall that the dilaton profile is $\phi(z)=\kappa_{N} z^{2}$ in the DiracBorn-Infeld action (nucleon and rho) and $2 \phi(z)$ in the Einstein-Hilbert action in the string frame (graviton).

A simultaneous fit to the rho-meson and nucleon radial trajectories (11) is achieved by choosing $\kappa_{N} \approx 350 \mathrm{MeV}$ as in the photoproduction analysis in [13], which gives $m_{N} \approx$ $990 \mathrm{MeV}$ and $m_{\rho} \approx 700 \mathrm{MeV}$, and therefore the degenerate glueball masses $m_{T}(0)=m_{S}(0)=\sqrt{2} m_{N} \approx 1386 \mathrm{MeV}$. 
The latters are to be compared to the lattice glueball masses $m_{2++} \approx 2150 \mathrm{MeV}$ and $m_{0++} \approx 1475 \mathrm{MeV}$ [26] (see also [27] for slightly heavier glueballs). The corresponding tensor gravitational radius (9) is

$$
\left\langle r_{G T}^{2}\right\rangle \approx(0.57 \mathrm{fm})^{2} .
$$

Conversely, if we fix the nucleon mass $m_{N}=940 \mathrm{MeV}$, then $\kappa_{N} \approx 330 \mathrm{MeV}$, the glueball masses are $m_{T}(0)=$ $m_{S}(0) \approx 1330 \mathrm{MeV}$, and the tensor gravitational radius (9) is slightly larger,

$$
\left\langle r_{G T}^{2}\right\rangle \approx(0.60 \mathrm{fm})^{2} .
$$

Note that the scalar dilaton field $T_{\mu}^{\mu}$ in (6) is characterized by the scalar form factor $A_{S}(k)\left(S=0^{++}\right.$glueball $)$with an equal mass radius in the strict holographic limit, i.e., $\left\langle r_{G S}^{2}\right\rangle=\left\langle r_{G T}^{2}\right\rangle$. The scalar radius is slightly larger at finite $1 / N_{c}$ [see (22) below].

\section{Comparison to lattice results}

In the lattice QCD calculation of the gravitational form factors [28], they use the decomposition of the energymomentum form factor in a nucleon state which is traceless and nonconserved

$$
\begin{aligned}
\left\langle p_{2}\left|T_{L}^{\mu \nu}(0)\right| p_{1}\right\rangle & \\
= & \bar{u}\left(p_{2}\right)\left(A(k) \gamma^{[\mu} p^{\nu]}+B(k) \frac{i p^{[\mu} \sigma^{\nu] \alpha} k_{\alpha}}{2 m_{N}}+C(k) \frac{k^{[\mu} k^{\nu]}}{m_{N}}\right) \\
& \times u\left(p_{1}\right),
\end{aligned}
$$

with $a_{[\mu} b_{\nu]}=\frac{1}{2}\left(a_{\mu} b_{\nu}+a_{\nu} b_{\mu}\right)-\frac{1}{4} \eta_{\mu \nu} a_{\alpha} b^{\alpha}$. Equation (14) cannot be probed directly by a metric fluctuation in bulk, since the latter couples only to the conserved energymomentum tensor. However, the invariant form factors are those of the conserved energy-momentum tensor, so they follow from the latter.

In Fig. 1 we compare the harmonic number result for $A(K)$ in (5) (solid curves) to the gluon lattice results (red dots). Since the holographic construction does not fix $A(0)$, we fixed it to $A(0)=0.58$ from the lattice data. The upper green-solid curve is fixed by the Regge mass $m_{T}(0)=$ $1.386 \mathrm{GeV}$, and the lower blue-solid curve is fixed by the Regge mass $m_{T}(0)=1.330 \mathrm{GeV}$. The dashed-purple curve is the dipole approximation

$$
A(K) \approx \frac{A(0)}{\left(1+\frac{K^{2}}{\tilde{m}_{T}^{2}}\right)^{2}}
$$

with $\tilde{m}_{T}=1.124 \mathrm{GeV}$. Throughout and for simplicity, we will use $\tilde{m}_{T, S}$ for the tensor and scalar parametric masses in the approximate but accurate dipole fit such as (15) to the holographic result (5). The latter resums the

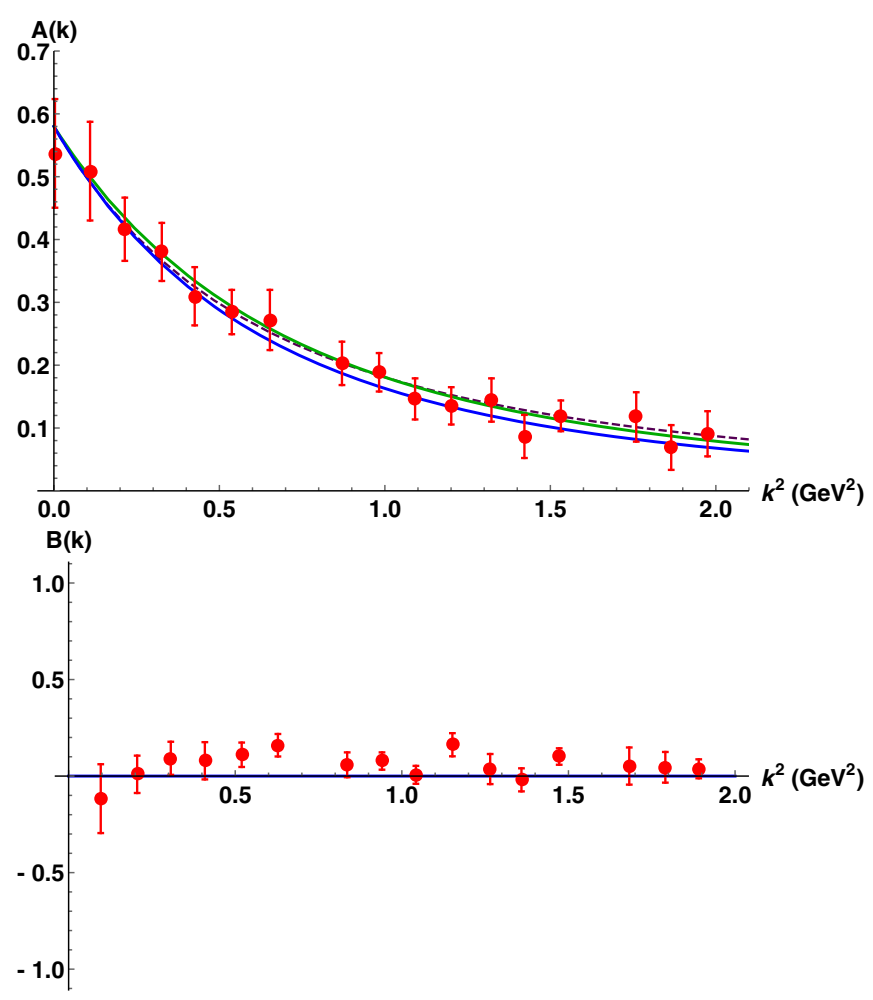

FIG. 1. Holographic nucleon gravitational form factors: $A(k)$ [blue-solid and green-solid curves (5) and purple-dashed curve (15)] and $B(k)$ (blue-solid curve) [13], versus the unquenched gluon lattice results (red dots) [28]. See text.

unapproximated tensor and scalar glueball Regge masses $m_{T, S}(n)$ in (10). The reported gluon lattice data are shown in red dots and are well fitted by a similar dipole with $\tilde{m}_{T, \text { lattice }}=1.13 \mathrm{GeV}$ which is undistinguishable from the dashed curve in this mass range [28]. $A(0)=0.58<1$ reflects on the gluon fraction assigned to the nucleon mass, fixed on the lattice but not in holography. We note that a dipole ansatz was originally used in [29] to describe $J / \Psi$ production through two massive gluons close to threshold.

The approximate dipole form (15) from both holography and the lattice suggests a tensor radius

$$
\left\langle r_{G T}^{2}\right\rangle \approx \frac{12}{\tilde{m}_{T}^{2}} \approx(0.62 \mathrm{fm})^{2},
$$

which is compatible with (12) and (13). It is comforting that in both our case and the gluon lattice case, $B(K)$ is consistent with zero. In holography, this is explained by the absence of a tensor coupling to a bulk Dirac fermion.

Equation (5) and its approximate dipole form (15) resum the infinite tower of monopoles stemming from the full $T=2^{++}$radial Regge trajectory in the dual limit of large $N_{c}$ and strong coupling. It is rather surprising that this resummed form is comparable to the unquenched tensor form factor probed by the gluon lattice form factor. This is suggestive of two things: (i) the quark mixing in the tensor 
channel is weak; (ii) the resummed radial $T=2^{++}$Regge trajectory is not very sensitive to the lower tensor glueball mass.

To compare the $D$-term with the gluon lattice results, we fit the exact harmonic number results for both $A(K)$ and $A_{S}(K)$ following from (4), with dipoles

$$
\begin{aligned}
D(K) & =4 C(K) \approx-\frac{4 m_{N}^{2}}{3 K^{2}}\left[\frac{A(0)}{\left(1+\frac{K^{2}}{\tilde{m}_{T}^{2}}\right)^{2}}-\frac{A(0)}{\left(1+\frac{K^{2}}{\tilde{m}_{S}^{2}}\right)^{2}}\right] \\
& =-\frac{8 A(0)}{3}\left(\frac{m_{N}^{2}}{\tilde{m}_{S}^{2}}-\frac{m_{N}^{2}}{\tilde{m}_{T}^{2}}\right)\left[\frac{1+\frac{K^{2}}{4}\left(\frac{1}{\tilde{\tilde{m}}_{S}^{2}}+\frac{1}{\tilde{m}_{T}^{2}}\right)}{\left(1+\frac{K^{2}}{\tilde{m}_{T}^{2}}\right)^{2}\left(1+\frac{K^{2}}{\tilde{m}_{S}^{2}}\right)^{2}}\right] \\
& =D(0)\left[\frac{1+\frac{K^{2}}{4}\left(\frac{1}{\tilde{m}_{S}^{2}}+\frac{1}{\tilde{m}_{T}^{2}}\right)}{\left(1+\frac{K^{2}}{\tilde{m}_{T}^{2}}\right)^{2}\left(1+\frac{K^{2}}{\tilde{m}_{S}^{2}}\right)^{2}}\right]
\end{aligned}
$$

with $A(0)=0.58, D(0)<0$, and $\tilde{m}_{T} \approx 1.124 \mathrm{GeV}$. The scalar mass satisfies

$$
\frac{\tilde{m}_{S}^{2}}{\tilde{m}_{T}^{2}}=\left(1-\frac{3 D(0)}{8 A(0)} \frac{\tilde{m}_{T}^{2}}{\tilde{m}_{N}^{2}}\right)^{-1}
$$

and for a null $D$-term, it matches the tensor mass. The $D$-term falls faster than the $A$-term and asymptotes $D(K) \approx D(0)\left(\tilde{m}_{S}^{2} \tilde{m}_{T}^{2}\right) / K^{6}$, which is consistent with the hard QCD counting rules for the proton $D$-term [14,30-32], in sharp contrast to the hard QCD scattering rules for the pion $D$-term [33]. In Fig. 2 we show (17) as a solid-blue curve versus the gluon lattice data red dots, and the dipole lattice fit

$$
D_{L}(K) \approx-\frac{10}{\left(1+\frac{K^{2}}{m_{D}^{2}}\right)^{2}}
$$

with $m_{D}=0.48 \mathrm{GeV}$ as the dashed-purple curve. The holographic parameters in (17) are set to $D(0)=-4$ and $\left(\tilde{m}_{T}, \tilde{m}_{S}\right)=(1.124,1.00) \mathrm{GeV}$. Again, it is remarkable

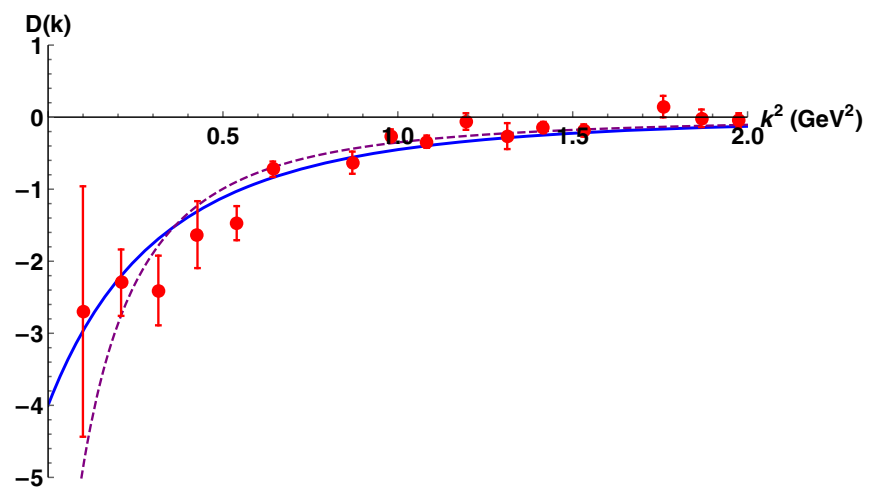

FIG. 2. Holographic nucleon gravitational form factors $D(k)=$ $4 C(k)$ [blue-solid curve (17)] versus the unquenched gluon lattice results (red dots) and lattice fit [purple-dashed curve (19)] [28]. See text. that the Reggeized holographic result fits rather well the reported lattice data. The latters are unquenched simulations, and one would have expected strong scalar-isoscalar quark mixing to the $0^{++}$glueball states: in particular to the light sigma meson with a mass of about $0.5 \mathrm{GeV}$ (although this state is rather broad).

The $D$-term (17) allows for the extraction of a new mass radius

$$
\left\langle r_{G D}^{2}\right\rangle=-\frac{42}{4}\left(\frac{1}{\tilde{m}_{S}^{2}}+\frac{1}{\tilde{m}_{T}^{2}}\right) \approx-(0.87 \mathrm{fm})^{2}
$$

which is larger in magnitude than the tensor mass radii (12), (13), and (16), but substantially smaller in magnitude than the lattice dipole estimate (21)

$$
\left\langle r_{G D}^{2}\right\rangle_{\text {lattice }}=-\frac{12}{m_{D}^{2}} \approx-(1.44 \mathrm{fm})^{2} .
$$

We note that since the $\left(\tilde{m}_{T}, \tilde{m}_{S}\right)=(1.124,1.00) \mathrm{GeV}$ fit to the lattice $D$-term implies $m_{S}(n) \neq m_{T}(n)$ at finite $1 / N_{c}$ in (10), it follows that the scalar and tensor radii are different, with the scalar radius

$$
\left\langle r_{G S}^{2}\right\rangle=\frac{12}{\tilde{m}_{S}^{2}} \approx(0.7 \mathrm{fm})^{2}
$$

slightly larger than the tensor radii (12), (13), and (16). Recall that the Reggeized scalar glueballs are sourced by $T_{\mu}^{\mu} \approx F^{2}$ (conformal anomaly) on the boundary, so the empirical identification of $\tilde{m}_{S}$ through the gluonic contribution $D(K)$ in Fig. 2 is justified. The value of $\tilde{m}_{T}$ is fixed solely by holography (5), (15) and is consistent with both the lattice gluonic tensor form factor $A(K)$ in Fig. 1, and the empirically extracted tensor form factor from the current GlueX data (see Fig. 4 below).

\section{PRESSURE AND SHEAR INSIDE THE PROTON}

We follow [18,34] (and references therein) and define the Fourier transform of the $D$-term

$$
\begin{aligned}
\tilde{D}(r) & =\int \frac{d^{3} K}{2 m_{N}(2 \pi)^{3}} e^{-i K \cdot r} D(K) \\
& =\frac{A(0) m_{N}}{6 \pi r}\left(e^{-\tilde{m}_{S} r}\left(2+\tilde{m}_{S} r\right)-e^{-\tilde{m}_{T} r}\left(2+\tilde{m}_{T} r\right)\right),
\end{aligned}
$$

where the approximate but numerically accurate dipole form (15), instead of the exact harmonic number form (5) for $A(K), A_{S}(K)$ is used for numerical convenience. The pressure $p(r)$ and shear $s(r)$ distributions in the proton, say in the Breit frame, can be expressed in terms of $\tilde{D}(r)$ as $[18,34]$ 


$$
\begin{aligned}
& p(r)=\frac{1}{3} \frac{1}{r^{2}} \frac{d}{d r}\left(r^{2} \frac{d}{d r} \tilde{D}(r)\right), \\
& s(r)=-\frac{r}{2} \frac{d}{d r}\left(\frac{1}{r} \frac{d}{d r} \tilde{D}(r)\right),
\end{aligned}
$$

as they capture the anisotropic spatial content of the energy momentum tensor

$$
T^{i j}(\vec{r})=\frac{1}{3} \delta^{i j} p(r)+\left(\hat{r}^{i} \hat{r}^{j}-\frac{1}{3} \delta^{i j}\right) s(r) .
$$

In Fig. 3 we show our holographic results for the radial pressure (top blue-solid curve) and radial shear (bottom red-solid curve) mass distributions inside the proton from (24) for $\tilde{m}_{S} / \tilde{m}_{T}=1 / 1.124$. The scale resolution is fixed at the nucleon mass. The red-dashed curve in the radial pressure is the estimate

$$
\frac{r^{2} p(r)}{A(0) m_{N} \tilde{m}_{T}} \approx \frac{\left(\tilde{m}_{T} r\right)^{2}}{200}\left(10 e^{-\tilde{m}_{T} r}-7 e^{-\tilde{m}_{S} r}\right) .
$$

The pressure distribution inside the proton is a delicate balance between the Reggeized scalar glueball $S=0^{++}$ attraction and the Reggeized tensor glueball $T=2^{++}$

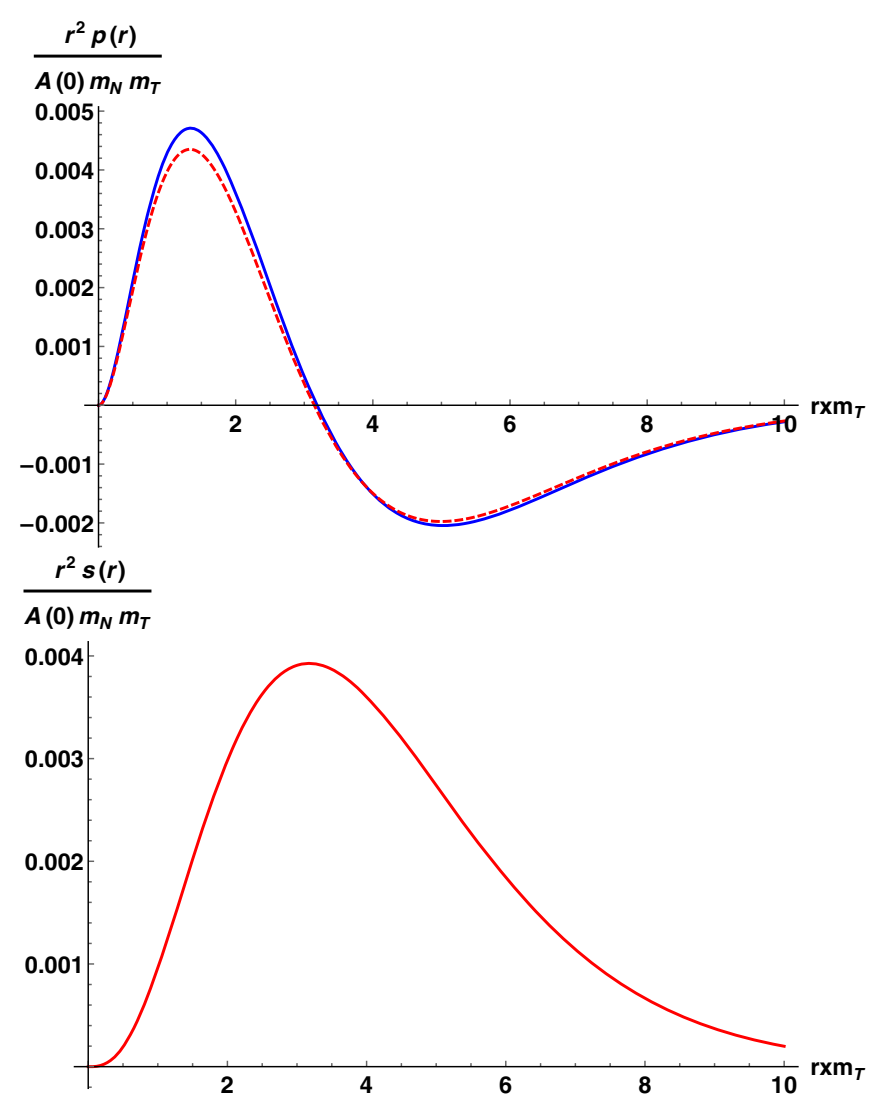

FIG. 3. Holographic gravitational pressure and shear inside the proton from (23) and (24) with $\tilde{m}_{S} / \tilde{m}_{T}=1 / 1.124$. See text. repulsion. The inside of the pressure is dominated by the repulsive tensor, while the outside of the pressure is dominated by the attractive scalar which ultimately keeps the proton together. The radial shear distribution inside the proton follows from a much more subtle difference, with the scalar and tensor roles reversed. The Reggeized scalar glueball $S=0^{++}$contributes positively to the shear, while the Reggeized tensor glueball $T=2^{++}$contributes negatively. The difference is in favor of the positive scalar contribution, with a net proton shear positive at all distances. Note that the scalar contribution to the shear follows from the traceless constraint on the spatial tensor contribution in (25) (quadrupole), which may explain the sign flip.

These results are comparable to the experimentally extracted quark contributions in [35] for both the pressure and the shear, and also some model calculations in [18,36] (and references therein). They are also comparable to the lattice QCD results reported in [37] at the higher scale resolution $\mu=2 \mathrm{GeV}$.

\section{TENSOR GRAVITATIONAL FORM FACTOR FROM GLUEX}

We now show that the threshold photoproduction of heavy vector mesons $V=J / \Psi, \Upsilon$, is solely driven by the tensor gravitational form factor $A(K)$ near threshold, which resums the tensor glueball $T=2^{++}$radial Regge trajectory. Way above threshold, the same process is dominated by the Reggeized form of this form factor following the resummation of the higher spin- $j$ Regge trajectories leading to the strong Pomeron exchange [13]. The $A$-form factor is measurable modulo a minimal kinematic assumption which involves the universal coupling of the graviton in bulk. It gives access to not only the proton gluon mass radius but also the $D$-term and therefore the gluon shear and pressure distributions inside the proton using holography.

\section{A. Gluon contribution to the proton mass and GlueX}

In QCD the energy-momentum tensor receives contributions from both the quarks and the gluons. Its forward matrix element in a proton state is fixed by Poincaré symmetry

$$
\begin{aligned}
\left\langle P\left|T_{G+Q}^{\mu \nu}\right| P\right\rangle & =2\left(A_{S G}(0)+A_{S Q}(0)=A_{S}(0)=A(0)\right) P^{\mu} P^{\nu} \\
& \equiv 2 P^{\mu} P^{\nu}
\end{aligned}
$$

with each of the gluon and quark contributions to the scalar form factor (6) fixed

$$
\begin{aligned}
& A_{S G}(0)=\frac{\left\langle P\left|-b g^{2} F^{2} / 32 \pi^{2}\right| P\right\rangle}{2 m_{N}^{2}}, \\
& A_{S Q}(0)=\frac{\langle P|m \bar{\psi} \psi| P\rangle}{2 m_{N}^{2}}=\frac{\sigma_{\pi N}}{m_{N}}
\end{aligned}
$$


with $b=11 N_{c} / 3-2 N_{f} / 3$ (1-loop). The gluonic contribution is fixed by the conformal anomaly, and the quark contribution by the pion-nucleon sigma term $\sigma_{\pi N} \approx 50 \mathrm{MeV}$. Note that Poincare symmetry in (27) implies that the tensor glueball exchange and the scalar glueball exchange matches at threshold. They both probe the scale anomaly, since $A(0) \approx A_{S G}(0)$ modulo the pion-nucleon sigma term which is small, i.e., $\sigma_{\pi N} / m_{N} \approx 1 / 20$.

The differential cross section for photoproduction of a heavy meson $\gamma p \rightarrow V p$ with $V=J / \Psi, \Upsilon$ can be calculated using the same Witten diagrams as for the form factor, with the result [13]

$$
\begin{aligned}
\left(\frac{d \sigma}{d t}\right)= & \frac{e^{2}}{64 \pi\left(s-m_{N}^{2}\right)^{2}} \times\left[\frac{1}{2} \frac{\kappa^{2}}{g_{5}^{4}} \mathbb{V}_{h A A}^{2}\right] \\
& \times\left[\frac{A^{2}(K)}{4 m_{N}^{2}} \times F\left(s, t=-K^{2}, M_{V}, m_{N}\right) \times\left(2 K^{2}+8 m_{N}^{2}\right)\right] .
\end{aligned}
$$

Note that asymptotically $F(s, t) \approx s^{4}$ which implies that the corresponding scattering amplitude is $\mathcal{A}(s, t) \approx s^{2}$, which is the signature of a $T=2^{++}$as a graviton exchange. This growth is tamed by a $j$-spin Reggeization giving rise to the strong Pomeron exchange at large $\sqrt{s}$. Since the combination

$$
\left[\frac{1}{2} \frac{\kappa^{2}}{g_{5}^{4}} \mathbb{V}_{h A A}^{2}\right] \rightarrow \frac{1}{2} \frac{4 \pi^{2} / N_{c}^{2}}{\left(12 \pi^{2} / N_{c}\right)^{2}} \mathbb{V}_{h A A}^{2}
$$

is nonuniversal, it is unfortunately not possible to extract the value of $A(0) \approx A_{S G}(0)$ from the interpolated differential cross section at threshold. It is, however, possible to do more and extract the full tensor form factor as we have originally suggested and shown in [13]. We now recall how.

\section{B. Extracting the tensor gravitational form factor from GlueX}

The recently reported GlueX data allow for the extraction of the tensor part of the gravitational form factor $A(K)$ modulo the kinematical function $F(s, t)$. The latter is fixed by the graviton coupling in bulk to a spin $1^{--}$flavor gauge field, which is universal. With this in mind, Eq. (29) suggests to use the empirical ratio of differential cross sections measured by the GlueX Collaboration [8] to extract $A(K)$. In Fig. 4 we show this empirical ratio $A(\Delta t) / A\left(\Delta t_{\min }\right)$ normalized by the first or minimal data point, with $\Delta t=\left(-\left(t-t_{\min }\right)\right)^{\frac{1}{2}}$ and $\Delta t_{\min }=\sqrt{0.075}$ versus $\Delta t^{2}$ in $\mathrm{GeV}^{2}$ [13].

$$
\begin{aligned}
& \frac{A(\Delta t)^{2}}{A\left(\Delta t_{\min }\right)^{2}} \\
& =\left(\frac{F\left(s, t=t_{\min }, M_{V}, m_{N}\right)\left(-2 t_{\min }+8 m_{N}^{2}\right)}{F\left(s, t=-K^{2}, M_{V}, m_{N}\right)\left(2 K^{2}+8 m_{N}^{2}\right)}\right) \frac{\left(\frac{d \sigma}{d t}\right)}{\left(\frac{d \sigma}{d t}\right)_{\min }} .
\end{aligned}
$$



FIG. 4. Extraction of the gravitational form factor $A\left(\sqrt{-\left(t-t_{\min }\right)}\right)$ [normalized by $A(\sqrt{0.075})$ ] from the recent GlueX data (crosses) [8]. The blue-solid line is the holographic tensor gravitational form factor (15) with $m_{T}=1.124 \mathrm{GeV}$ and $m_{J / \psi}=3.10 \mathrm{GeV}$ [13]. The red-dashed line is the lattice tensor gravitational form factor (15) with $m_{\text {Tlattice }}=1.13 \mathrm{GeV}$ [28].

The holographic result is shown as the blue-solid line and the lattice result as the red-dashed line. The $t$-dependence in $F(s, t)$ in the range currently probed by GlueX is weak, making the ratio of the differential cross sections commensurate with the squared tensor form factor. The agreement of the extracted GlueX form factor with both calculations suggests that the gluonic part of the shear and pressure distributions as well as the mass radius is well captured by the holographic construction, and now measured. The empirical errors for the ratio have been added in quadrature using the GlueX data.

The consistency of the GlueX data with the holographic and lattice estimated curves using the dipole form (15) suggests a common tensor mass radius (16) or

$$
\left\langle r_{\text {GlueX }}^{2}\right\rangle \approx(0.62 \mathrm{fm})^{2}
$$

A recent analysis of the same data at threshold extracted $\left\langle r_{G}^{2}\right\rangle=(0.55 \pm 0.03 \mathrm{fm})^{2}$ [38] which is slightly smaller than the mass radius from the global extraction (32), but close to the holographic threshold value (12) from the exact form factor (5) using the simultaneous fit to the nucleon and rho meson radial Regge trajectories. Finally, also a recent analysis of the combined data for photoproduction of vector mesons yields $\left\langle r_{G}^{2}\right\rangle \approx(0.64 \pm 0.03 \mathrm{fm})^{2}$ [39], which is comparable to (16) and (32).

\section{CONCLUSIONS}

The gluonic content of the three gravitational form factors is accessible from holographic QCD in the double limit of large $N_{c}$ and strong coupling. The $A$-term is dominated by the tensor $T=2^{++}$Reggeized radial glueball trajectories, while the $D$-term involves the difference between the tensor $T=2^{++}$and $S=0^{++}$Reggeized radial glueball trajectories, which are degenerate in the strict 
holographic limit (same anomalous dimension). This degeneracy is lifted in $1 / N_{c}$. The $B$-term vanishes in the absence of a tensor coupling to a Dirac fermion in bulk.

The holographic result for the $A$-term and $D$-term compares well with the unquenched lattice results suggesting that there is little quark mixing in both the $T=2^{++}$ and the $S=0^{++}$channels when they are fully Reggeized. This is a remarkable observation that can be further tested by carrying the lattice calculations in the unquenched limit. The $D$-term allows for the extraction of both the gluonic pressure and shear inside the proton in the holographic limit. We recall that the holographic scale in the form factors follows from the dilaton potential which is fixed by the nucleon and rho radial Regge trajectories.

The radial pressure distribution inside the proton follows from a delicate balance between the Reggeized tensor $T=$ $2^{++}$repulsion at short distances, and the attraction from the Reggeized scalar $S=0^{++}$at larger distances. The latter is what ultimately keeps the proton together. The roles are reversed in the radial distribution of the shear inside the proton, but the trade is much more subtle. The net proton shear stems from the difference between the scalar sheer due to the $S=0^{++}$glueball exchange which is positive and the tensor sheer due to the $T=2^{++}$glueball exchange which is negative. The difference is net positive at all distances.
The recently reported GlueX data can be used to extract the full tensor form factor or $A$-term modulo its threshold normalization. The tensor mass radius extracted from the GlueX data $\left\langle r_{\text {GlueX }}^{2}\right\rangle \approx(0.62 \mathrm{fm})^{2}$ using a global fit is compatible with $\left\langle r_{G T}^{2}\right\rangle \approx(0.57-0.60 \mathrm{fm})^{2}$ from the holographic threshold result. A comparison of the holographic $D$-term to the lattice data suggests a slightly larger scalar mass radius $\left\langle r_{G S}^{2}\right\rangle \approx(0.7 \mathrm{fm})^{2}$, among other things. A larger scalar radius was also recently noted in [40].

Finally, since the holographic $A$-term and $D$-term are related, and the $A$-term is accessible from the GlueX data over a broad range of momenta, we conclude that the GlueX data allow us to glean to the gluonic pressure and shear inside the proton via holography.

\section{ACKNOWLEDGMENTS}

We thank Xiang-dong Ji, Zein-Eddine Meziani, and Lubomir Pentchev for discussion. K. M. is supported by the U.S. Department of Energy, Office of Science, Office of Nuclear Physics, Contract No. DE-AC02-06CH11357, and an LDRD initiative at Argonne National Laboratory under Project No. 2020-0020. I.Z. is supported by the Office of Science, U.S. Department of Energy under Contract No. DE-FG-88ER40388.
[1] F. Wilczek, Origins of mass, Central Eur. J. Phys. 10, 1021 (2012).

[2] C. D. Roberts, On mass and matter, arXiv:2101.08340.

[3] E. Shuryak, Lectures on nonperturbative QCD (Nonperturbative Topological phenomena in QCD and related theories), arXiv:1812.01509.

[4] I. Zahed, Mass sum rule of hadrons in the QCD instanton vacuum, arXiv:2102.08191.

[5] D. B. Leinweber, Visualizations of the QCD vacuum, in Workshop on Light-Cone QCD and Nonperturbative Hadron Physics, edited by A. W. Schreiber and A. G. Williams (World scientific, 2000), pp. 138-143 [arXiv:hep-lat/0004025].

[6] J. C. Biddle, W. Kamleh, and D. B. Leinweber, Visualization of center vortex structure, Phys. Rev. D 102, 034504 (2020).

[7] K. Hafidi, S. Joosten, Z. E. Meziani, and J. W. Qiu, Production of charmonium at threshold in Hall A and $\mathrm{C}$ at Jefferson Lab Few-Body Syst. 58, 141 (2017).

[8] A. Ali et al. (GlueX Collaboration), First Measurement of Near-Threshold J/ $\psi$ Exclusive Photoproduction Off the Proton, Phys. Rev. Lett. 123, 072001 (2019).

[9] Z.-E. Meziani and S. Joosten, Origin of the proton mass? Heavy quarkonium production at threshold from jefferson lab to an electron ion collider, in Probing Nucleons and Nuclei in High Energy Collisions: Dedicated to the Physics of the Electron Ion Collider (2020), pp. 234-237.
[10] D. P. Anderle et al., Electron-ion collider in China, arXiv:2102.09222.

[11] M. S. Costa, M. Djurić, and N. Evans, Vector meson production at low $\mathrm{x}$ from gauge/gravity duality, J. High Energy Phys. 09 (2013) 084.

[12] C. H. Lee, H.-Y. Ryu, and I. Zahed, Diffractive vector photoproduction using holographic QCD, Phys. Rev. D 98, 056006 (2018).

[13] K. A. Mamo and I. Zahed, Diffractive photoproduction of $J / \psi$ and $\Upsilon$ using holographic QCD: Gravitational form factors and GPD of gluons in the proton, Phys. Rev. D 101, 086003 (2020).

[14] Y. Hatta and D.-L. Yang, Holographic $J / \psi$ production near threshold and the proton mass problem, Phys. Rev. D 98, 074003 (2018).

[15] I. Yu. Kobzarev and L. B. Okun, Gravitational interaction of fermions, Zh. Eksp. Teor. Fiz. 43, 1904 (1962).

[16] H. Pagels, Energy-momentum structure form factors of particles, Phys. Rev. 144, 1250 (1966).

[17] P. Carruthers, Broken scale invariance, Phys. Rev. D 2, 2265 (1970).

[18] M. V. Polyakov and P. Schweitzer, Forces inside hadrons: Pressure, surface tension, mechanical radius, and all that, Int. J. Mod. Phys. A 33, 1830025 (2018).

[19] H. Nastase, Introduction to AdS-CFT, arXiv:0712.0689. 
[20] I. Kanitscheider, K. Skenderis, and M. Taylor, Precision holography for non-conformal branes, J. High Energy Phys. 09 (2008) 094.

[21] Z. Abidin and C. E. Carlson, Nucleon electromagnetic and gravitational form factors from holography, Phys. Rev. D 79, 115003 (2009).

[22] S. S. Gubser, A. Nellore, S. S. Pufu, and F. D. Rocha, Thermodynamics and Bulk Viscosity of Approximate Black Hole Duals to Finite Temperature Quantum Chromodynamics, Phys. Rev. Lett. 101, 131601 (2008).

[23] H. Forkel, Holographic glueball structure, Phys. Rev. D 78, 025001 (2008).

[24] P. Colangelo, F. De Fazio, F. Jugeau, and S. Nicotri, Investigating AdS/QCD duality through scalar glueball correlators, Int. J. Mod. Phys. A 24, 4177 (2009).

[25] H. Boschi-Filho, N. R. F. Braga, F. Jugeau, and M. A. C. Torres, Anomalous dimensions and scalar glueball spectroscopy in AdS/QCD, Eur. Phys. J. C 73, 2540 (2013).

[26] H. B. Meyer, Glueball regge trajectories, Other thesis, 2004, arXiv:hep-lat/0508002.

[27] B. Lucini and M. Teper, SU(N) gauge theories in fourdimensions: Exploring the approach to $\mathrm{N}=$ infinity, J. High Energy Phys. 06 (2001) 050.

[28] P. E. Shanahan and W. Detmold, Gluon gravitational form factors of the nucleon and the pion from lattice QCD, Phys. Rev. D 99, 014511 (2019).

[29] L. Frankfurt and M. Strikman, Two-gluon form factor of the nucleon and $\mathrm{j} / \psi$ photoproduction, Phys. Rev. D 66, 031502 (2002).
[30] K. Tanaka, Operator relations for gravitational form factors of a spin-0 hadron, Phys. Rev. D 98, 034009 (2018).

[31] Y. Hatta and M. Strikman, $\phi$-meson lepto-production near threshold and the strangeness $D$-term, arXiv:2102 .12631 .

[32] X.-B. Tong, J.-P. Ma, and F. Yuan, Gluon gravitational form factors at large momentum transfer, arXiv:2101.02395.

[33] E. Shuryak and I. Zahed, Nonperturbative quark-antiquark interactions in mesonic form factors, Phys. Rev. D 103, 054028 (2021).

[34] M. V. Polyakov, Generalized parton distributions and strong forces inside nucleons and nuclei, Phys. Lett. B 555, 57 (2003).

[35] V. D. Burkert, L. Elouadrhiri, and F. X. Girod, The pressure distribution inside the proton, Nature (London) 557, 396 (2018).

[36] J. Yu. Panteleeva and M. V. Polyakov, Forces inside the nucleon on the light front from 3D Breit frame force distributions: Abel tomography case, arXiv:2102.10902.

[37] P. E. Shanahan and W. Detmold, Pressure Distribution and Shear Forces Inside the Proton, Phys. Rev. Lett. 122, 072003 (2019).

[38] D. E. Kharzeev, The mass radius of the proton, arXiv: 2102.00110

[39] R. Wang, W. Kou, and X. Chen, Extraction of the proton mass radius from the vector meson photoproductions near thresholds, arXiv:2102.01610 [Phys. Rev. D (to be published)].

[40] X. Ji, Proton mass decomposition: Naturalness and interpretations, Front. Phys. 16, 64601 (2021), arXiv:2102.07830. 